\title{
MicroRNA-1271-5p inhibits the tumorigenesis of ovarian cancer through targeting E2F5 and negatively regulates the mTOR signaling pathway
}

\section{Qin Li}

The First People's Hospital of Changzhou

Junyu Shi

The First People's Hospital of Changzhou

Xiaoli Xu ( $\sim$ qhmyp0909@163.com)

Department of Obstetrics and Gynecology, The Third Affiliated Hospital of Soochow University

\section{Research}

Keywords: miR-1271-5p, E2F5, ovarian cancer, mTOR

Posted Date: March 19th, 2020

DOl: https://doi.org/10.21203/rs.3.rs-17976/v1

License: (c) (i) This work is licensed under a Creative Commons Attribution 4.0 International License.

Read Full License 


\section{Abstract}

Background: MicroRNA-1271-5p (miR-1271-5p) has been reported to participate in the progression of many malignancies. However, the molecular mechanism of miR-1271-5p still remains vague in ovarian cancer (OC). Therefore, we explored the effect of miR-1271-5p in the development of OC in present study.

Methods: We measured the miR-1271-5p expression via qRT-PCR assay. Western blot analysis was employed to examine protein expression. Then, the functional mechanism of miR-1271-5p was analyzed by MTT, Transwell and dual luciferase assays.

Results: Downregulation of miR-1271-5p was found in OC, which can predict worse prognosis in OC patients. Further, miR-1271-5p directly targets E2F5 in OC. And miR-1271-5p restrained the proliferation, migration and invasion of $\mathrm{OC}$ cells via targeting E2F5. Additionally, upregulation of E2F5 was observed in OC, which predicted unfavorable prognosis in OC patients. Besides that, miR-1271-5p suppressed EMT and mTOR pathway in OC.

Conclusion: MiR-1271-5p inhibited the tumorigenesis of OC through targeting E2F5 and negatively regulated the mTOR signaling pathway.

\section{Introduction}

Ovarian cancer $(\mathrm{OC})$ is a kind of common cancer in female reproductive organs, ranking third only to cervical cancer and uterine cancer [1]. But the death caused by $\mathrm{OC}$ is the first in all kinds of gynecological tumors, posing a serious threat to women's life [2]. At present, the etiology of $\mathrm{OC}$ is unclear, which may be related to age, fertility, blood type, mental factors and environment [3]. Moreover, the treatment of OC is different due to different pathological types, and operation combined with chemotherapy is usually used to treat OC $[4,5]$. In addition, the five-year survival rate of OC patients is very low, only 25-30\% [6]. However, if it is found early, $90 \%$ of the OC patients will survive; Later, when cancer cells spread to the ovary, the survival rate is less than $30 \%$ [7]. Hence, early diagnosis and early treatment are very significant to enhance survival rate of $\mathrm{OC}$ patients.

In recent years, microRNAs (miRNAs) have been paid more and more attention because of their specific function in various cancers and disease. Attention is mainly focused on their inhibitory effect on some genes expression [8]. Additionally, many miRNAs have been reported to regulate the progress of OC. For example, miR-365 inhibited OC progression by targeting Wnt5a [9]. And miR-1 inhibited cell migration and proliferation in OC through c-Met pathway [10]. But miR-205 accelerated cell invasion by repressing TCF21 in human OC [11]. And miR-216a facilitated the metastasis and EMT of OC by suppressing the PTEN/AKT pathway [12]. These studies imply that miRNA can function as a biomarker and target in OC. Especially, miR-1271 has been found to express abnormally and show different functions in human cancers. MiR-1271 has been proposed to block breast cancer progression by regulating circ-ABCB10 [13]. Moreover, miR-1271 had been found to function as an inhibitor for metastasis and EMT in human HCC [14]. And the suppressive effect of miR-1271 was also detected in colorectal cancer cells [15]. In addition, 
expression and role of miR-1271 was reported to affect the pathogenesis of osteosarcoma [16]. However, it was reported that miR-1271 promoted non-small-cell lung cancer cell proliferation and invasion [17]. Previous studies suggested that miR-1271 displayed different roles in different human cancers. And these studies have stimulated our desire to determine miR-1271 role in OC.

In addition, it has been revealed that E2F transcription factor 5 (E2F5) can promote cell proliferation in hepatocellular carcinoma cells [18]. Besides, upregulation of E2F5 had been identified in some human cancers including breast cancer [19], gastric cancer [20] and glioblastoma [21]. What's more, miR-98 had been found to delay skeletal muscle differentiation by downregulating E2F5 [22]. And E2F5 had been identified as an independent prognostic factor in esophageal squamous cell carcinoma [23]. However, the specific effect of E2F5 in OC remains unclear and need to be explored.

Here, the abnormal expression of miR-1271-5p was observed in OC. And the functions of miR-1271-5p were also analyzed in the development of OC. The relationship between miR-1271-5p and E2F5 was also confirmed in OC. In addition, the effect of miR-1271-5p on EMT and mTOR signaling pathway was analyzed in this study.

\section{Materials And Methods}

\section{Clinical samples}

Forty-five human OC tissues and normal ovary tissues were acquired from The Third Affiliated Hospital of Soochow University (The First People's Hospital of Changzhou). Written informed consents were collected from all participators. All OC patients only received surgical treatment. This research was approved by the Institutional Ethics Committee of The Third Affiliated Hospital of Soochow University (The First People's Hospital of Changzhou).

\section{Cell lines culture}

OC cells A2780, SKOV3, OVCAR and human normal ovarian epithelial cell line IOSE80 were purchased from Tumor Cell Bank of the Chinese Academy of Medical Science (Beijing, China). Then, RPMI-1640 medium with $10 \%$ fetal bovine serum (FBS) was used to incubate these cells at $37^{\circ} \mathrm{C}$ with $5 \% \mathrm{CO}_{2}$.

\section{Cell transfection}

MiR-1271-5p plasmid and negative control (NC) or miR-1271-5p mimics or inhibitor were obtained from Ribobio (Guangzhou, China). Lipofectamine 2000 (Invitrogen, Carlsbad, USA) was employed to transfected then into SKOV3 cells.

\section{Quantitative RT-PCR}

Total RNA was obtained by using TRIzol reagent (Invitrogen, Carlsbad, USA). And PrimeScript RT reagent kit (TaKaRa, Dalian, China) was used to obtain cDNA. Quantitative RT-PCR was conducted using SYBR 
Premix Ex Taq (TaKaRa, USA). MiR-1271-5p or E2F5 was normalized to U6 or GAPDH. The $2^{-\triangle} \triangle \mathrm{ct}$ method was selected to analyze their expressions. The forward primer for miR-1271-5p was 5'-CTT GGC ACC TAG CAA GCA CTC A-3', and the reverse primer was 5'-TAT GGT TGT TCT CCT CTC TGT CTC-3'. The internal control for miR-1271-5p was GAPDH (forward, 5'-CGG AGT CAA CGG ATT TGG TCG TAT-3'; reverse, 5'-AGC CTT CTC CAT GGT GGT GAA GAC-3'). The primers for E2F5 were 5'-CCT GTT CCC CCA CCT GAT G-3 (forward) and 5'-TTT CTG TGG AGT CAC TGG AGT CA-3' (reverse). The internal control was U6 (forward, 5'-CTC GCT TCG GCA GCA CA-3'; reverse, 5'-AAC GCT TCA CGA ATT TGC GT-3').

\section{MTT assay}

The transfected cells were incubated in $96-$ well plates for $24,48,72$ and $96 \mathrm{~h}$. Next, $20 \mu \mathrm{L}$ MTT solutions (Thermo Fisher Scientific, Inc.) was used to incubate the cells for $4 \mathrm{~h}$. After that, we terminated the incubation and discarded culture supernatant. Finally, OD value $(490 \mathrm{~nm})$ was detected using a spectrophotometer.

\section{Transwell assays}

Transwell chambers (8- $\mu \mathrm{m}$ pore size membranes) were employed to perform cell migration and invasion assays. Cell invasion was detected in the upper chambers (8- $\mu \mathrm{m}$ pore size membranes) with matrigel (BD Biosciences, USA). The lower chamber was added with $10 \%$ FBS. Transfected SKOV3 and OVCAR cells were incubated for $24 \mathrm{~h}$ at $37^{\circ} \mathrm{C}$ with $5 \% \mathrm{CO}_{2}$. Methanol and crystal violet were applied to fix and stain the invasive cells. Cell migration was detected without matrigel. Finally, the number of removed cells was measured by a microscope.

\section{Dual Luciferase Assay}

The pmirGLO luciferase vector (Promega, Madison, USA) containing wild or mutant type of 3'-UTR of E2F5 was transfected into SKOV3 and OVCAR cells with miR-1271-5p mimics. The luciferase activity was observed using dual luciferase assay system (Promega, USA).

\section{Western blot analysis}

Protein was obtained and separated by RIPA lysis buffer and 10\% SDS-PAGE. Then, the protein was blocked with $5 \%$ non-fat milk and transferred in PVDF membranes. Next, the membranes were incubated with vimentin, N-cadherin, E-cadherin, mTOR, and GAPDH primary antibodies (1:1000; Abcam, USA) overnight at $4^{\circ} \mathrm{C}$. After washing, secondary antibody (1:2000; Abcam, USA) was continued to incubate the protein for $2 \mathrm{~h}$. Finally, protein expression was observed using ECL (ECL, Pierce).

\section{Statistical analysis}

Data were analyzed by Graphpad Prism 6 and SPSS 19.0 and presented as mean \pm SD. Student's t test or Tukey's one-way ANOVA was selected to calculate the differences between groups. Kaplan-Meier analysis with log-rank test was applied to analyze the survival differences. $P<0.05$ indicates statistical difference. 


\section{Results}

\section{Decreased expression of miR-1271-5p was detected in OC.}

Primarily, miR-1271-5p expression was measured in OC tissues. The qRT-PCR assay suggested that miR1271-5p expression was apparently declined in OC tissues contrast to normal tissues (Figure 1A). In addition, low miR-1271-5p expression was associated with FIGO stage $(P=0.035)$ and lymph node metastasis $(P=0.007)$ in OC patients (Table 1). Therefore, we considered that miR-1271-5p was downregulated in $\mathrm{OC}$ that might involve in the tumorigenesis of $\mathrm{OC}$. In addition, Kaplan-Meier survival curve indicated that low miR-1271-5p expression was associated with infaust prognosis in OC patients ( $P$ $=0.0051$, Figure 1B). According to above result, aberrant expression of miR-1271-5p might relate to the prognosis of $\mathrm{OC}$ patient.

\section{MiR-1271-5p restrained cell proliferation, migration and invasion in OC.}

Then, miR-1271-5p expression level was examined in A2780, SKOV3, OVCAR and IOSE80 cell lines to further investigate its effect in OC. Consistent with the results of OC tissues, miR-1271-5p expression was apparently declined in A2780, SKOV3 and OVCAR cells contrast to IOSE80 cells (Figure 2A). Then we found that miR-1271-5p expression was distantly enhanced by miR-1271-5p mimics and was reduced by miR-1271-5p inhibitor detected by qRT-PCR (Figure 2B). Furthermore, miR-1271-5p overexpression repressed SKOV3 and OVCAR cell proliferation, whereas miR-1271-5p downregulation showed opposite effect on cell proliferation (Figure 2C, 2D). Besides that, the results of Transwell assay displayed that miR1271-5p mimics repressed cell migration, when miR-1271-5p inhibitor significantly stimulated SKOV3 and OVCAR cell migration (Figure 2E). Meanwhile, the same result of miR-1271-5p was also identified for OC cell invasion (Figure 2F). Taken together, miR-1271-5p overexpression suppresses cell proliferation, migration and invasion in OC.

\section{E2F5 was a direct target of miR-1271-5p in OC cells.}

E2F5 was predicted to have a binding site with miR-1271-5p in TargetScan database (http://www.targetscan.org/, Figure 3A). Luciferase reporter assay suggested that the luciferase activity was reduced in SKOV3 and OVCAR cells with miR-1271-5p mimics and E2F5-Wt vector (Figure 3B). Additionally, we also found that E2F5 expression was negatively correlated with miR-1271-5p expression in OC tissues (Pख0.0001, $R^{2}=0.5541$; Figure $3 C$ ). Besides that, we found that E2F5 was downregulated by miR-1271-5p mimics (Figure 3D) and upregulated by miR-1271-5p inhibitor in SKOV3 and OVCAR cells (Figure 3E). Hence, E2F5 was verified as a direct target of miR-1271-5p and had negative correlation with miR-1271-5p expression.

\section{Upregulation of E2F5 was identified in OC tissues.}

Next, alternation of E2F5 expression was identified in OC tissues. The qRT-PCR experiment showed that E2F5 expression was increased in the OC tissue (Figure 4A). And upregulation of E2F5 was also observed 
in A2780, SKOV3, OVCAR cell lines in contrast to IOSE80 cells (Figure 4B). Besides that, high E2F5 expression predicted adverse prognosis in OC patients $(P=0.0092$, Figure $4 C)$. Therefore, E2F5 was considered to influence the development of OC.

\section{MiR-1271-5p negatively regulated EMT and mTOR pathway in OC.}

In addition, whether miR-1271-5p regulates EMT and mTOR pathway was explored in OC. Western blot analysis showed that miR-1271-5p overexpression promoted the expression of E-cadherin and suppressed $\mathrm{N}$-cadherin and Vimentin expressions (Figure 5A). Inversely, downregulation of miR-1271-5p exerted opposite effect on the expression of these makers (Figure 5B). Thus, we considered that miR1271-5p could suppress cell metastasis by regulating EMT. Besides that, we found that upregulation of miR-1271-5p reduced $p-m T O R$ expression, but no change was found for mTOR expression level (Figure $5 A$ ). On the contrary, downregulation of miR-1271-5p enhanced p-mTOR expression level (Figure 5B). In brief, miR-1271-5p was found to negatively regulate EMT and mTOR pathway in OC.

\section{Discussion}

The alternation of miRNAs expression has been demonstrated to participate in pathogenesis of human cancers including OC [24], [25]. In this study, miR-1271-5p expression was found to be decreased in OC. And downregulation of miR-1271-5p can predict worse prognosis in OC patients. Functionally, miR-1271$5 p$ repressed the proliferation, migration and invasion of OC cells via regulating E2F5 and negatively regulated the mTOR signaling pathway in OC. Therefore, miR-1271-5p was verified as a tumor suppressor in OC.

Recently, it has been identified that miR-1271 is a suppressive miRNA in several human malignant tumors. MiR-1271 was found to repress cellular proliferation in hepatocellular carcinoma [26] which is consistent with our results in OC. And miR-1271 also suppressed migration, invasion and EMT in pancreatic cancer cells [27]. Here, the same effect of miR-1271-5p on cell migration, invasion and EMT was found in $\mathrm{OC}$ as well. More importantly, Liu et al found that miR-1271 restrained tumor growth in OC [28] which is consistent with our findings. Besides that, miR-1271 was also reported to negatively regulate mTOR signaling in pancreatic cancer [29] as well as in OC in current research. In addition, low expression of miR-1271 had been reported to predict poor prognosis of patients with neuroglioma [30] as well as our findings. All these studies supported our conclusion about the role of miR-1271-5p in OC again. Furthermore, E2F5 was identified as a direct target of miR-1271-5p in OC.

E2F5 was reported to affect DNA synthesis, initiation of replication and cell-cycle [31]. Zhao et al. found that E2F5 functioned as an oncogene with copy number gain in prostate cancer [32] and we also found the carcinogenesis of E2F5 in OC in this study. Moreover, upregulation of E2F5 has been observed in gastric cancer [33] and colorectal cancer [34]. And the upregulation of E2F5 was also identified in OC. Additionally, we also examined that high E2F5 expression was related to poor prognosis of OC. Same as our results, it was reported that upregulation of E2F5 resulted in a worse clinical outcome and poor prognosis of breast cancer [35]. Besides that, E2F5 had been verified as a direct target of several miRNAs 
and had negative association with their expression, such as miR-129-3p [36], miR-132 [37] and miR-613 [38]. In present research, miR-1271-5p directly targets E2F5 and inversely regulated E2F5 expression in OC. These results showed that miR-1271-5p suppressed the development of OC, at least in part, through inhibiting E2F5 expression.

\section{Conclusion}

In this study, decreased expression of miR-1271-5p was detected in OC tissues and can predict unfavorable prognosis in OC patient. Moreover, miR-1271-5p inhibited the development of OC through regulating E2F5 and negatively regulated the mTOR signaling pathway in OC. These findings might be benefit for the diagnosis and therapy of OC.

\section{Declarations}

\section{Acknowledgements}

Not applicable.

\section{Funding}

Not applicable.

\section{Availability of data and materials}

The datasets used and/or analyzed during the present study are available from the corresponding author on reasonable request.

\section{Authors' contributions}

QL wrote the manuscript and analyzed the data. JS sorted out experimental data and performed the data analyses. XX contributed to the conception of the study. All authors read and approved the final manuscript.

\section{Ethics approval and consent to participate}

The study was approved by the Ethics Committee of The Third Affiliated Hospital of Soochow University (The First People's Hospital of Changzhou). Signed written informed consents were obtained from the patients and/or guardians.

\section{Consent for publication}

Not applicable.

\section{Competing interests}


The authors declare that they have no competing interests.

\section{References}

[1] Siegel RL, Miller KD, Jemal A. Cancer statistics, 2016. CA: a cancer journal for clinicians 2016;66:7-30.

[2] Jayson GC, Kohn EC, Kitchener HC, Ledermann JA. Ovarian cancer. Lancet 2014;384:1376-88.

[3] Permuth-Wey J, Sellers TA. Epidemiology of ovarian cancer. Methods in molecular biology 2009;472:413-37.

[4] Bristow RE. Surgical standards in the management of ovarian cancer. Current opinion in oncology 2000;12:474-80.

[5] Harries M, Gore M. Part II: chemotherapy for epithelial ovarian cancer-treatment of recurrent disease. The Lancet Oncology 2002;3:537-45.

[6] Waldron L, Haibe-Kains B, Culhane AC, Riester M, Ding J, Wang XV, et al. Comparative meta-analysis of prognostic gene signatures for late-stage ovarian cancer. Journal of the National Cancer Institute 2014;106.

[7] Liu J, Matulonis UA. New strategies in ovarian cancer: translating the molecular complexity of ovarian cancer into treatment advances. Clinical cancer research : an official journal of the American Association for Cancer Research 2014;20:5150-6.

[8] Calin GA, Croce CM. MicroRNA signatures in human cancers. Nature reviews Cancer 2006;6:857-66.

[9] Wang Y, Xu C, Wang Y, Zhang X. MicroRNA-365 inhibits ovarian cancer progression by targeting Wnt5a. American journal of cancer research 2017;7:1096-106.

[10] Qu W, Chen X, Wang J, Lv J, Yan D. MicroRNA-1 inhibits ovarian cancer cell proliferation and migration through c-Met pathway. Clinica chimica acta; international journal of clinical chemistry 2017;473:237-44.

[11] Wei J, Zhang L, Li J, Zhu S, Tai M, Mason CW, et al. MicroRNA-205 promotes cell invasion by repressing TCF21 in human ovarian cancer. Journal of ovarian research 2017;10:33.

[12] Liu H, Pan Y, Han X, Liu J, Li R. MicroRNA-216a promotes the metastasis and epithelial-mesenchymal transition of ovarian cancer by suppressing the PTEN/AKT pathway. OncoTargets and therapy 2017;10:2701-9.

[13] Liang HF, Zhang XZ, Liu BG, Jia GT, Li WL. Circular RNA circ-ABCB10 promotes breast cancer proliferation and progression through sponging miR-1271. American journal of cancer research 2017;7:1566-76. 
[14] Li C, Jiang Y, Miao R, Qu K, Zhang J, Liu C. MicroRNA-1271 functions as a metastasis and epithelialmesenchymal transition inhibitor in human HCC by targeting the PTP4A1/c-Src axis. International journal of oncology 2018;52:536-46.

[15] Li J, Xu J, Yan X, Jin K, Li W, Zhang R. Suppression of Capn4 by microRNA-1271 impedes the proliferation and invasion of colorectal cancer cells. Biomedicine \& pharmacotherapy = Biomedecine \& pharmacotherapie 2018;99:162-8.

[16] Lu G, Du L, Guo Y, Xing B, Lu J, Wei Y. Expression and role of microRNA-1271 in the pathogenesis of osteosarcoma. Experimental and therapeutic medicine 2018;15:1934-40.

[17] Wang Y, Xu L, Jiang L. miR-1271 promotes non-small-cell lung cancer cell proliferation and invasion via targeting HOXA5. Biochemical and biophysical research communications 2015;458:714-9.

[18] Sun J, Li H, Huo Q, Cui M, Ge C, Zhao F, et al. The transcription factor FOXN3 inhibits cell proliferation by downregulating E2F5 expression in hepatocellular carcinoma cells. Oncotarget 2016;7:43534-45.

[19] Li L, Wu C, Zhao Y. miRNA-34a enhances the sensitivity of gastric cancer cells to treatment with paclitaxel by targeting E2F5. Oncology letters 2017;13:4837-42.

[20] Xu H, Fei D, Zong S, Fan Z. MicroRNA-154 inhibits growth and invasion of breast cancer cells through targeting E2F5. American journal of translational research 2016;8:2620-30.

[21] Xu X, Cai N, Zhi T, Bao Z, Wang D, Liu Y, et al. MicroRNA-1179 inhibits glioblastoma cell proliferation and cell cycle progression via directly targeting E2F transcription factor 5 . American journal of cancer research 2017;7:1680-92.

[22] Kropp J, Degerny C, Morozova N, Pontis J, Harel-Bellan A, Polesskaya A. miR-98 delays skeletal muscle differentiation by down-regulating E2F5. The Biochemical journal 2015;466:85-93.

[23] Ishimoto T, Shiozaki A, Ichikawa D, Fujiwara H, Konishi H, Komatsu S, et al. E2F5 as an independent prognostic factor in esophageal squamous cell carcinoma. Anticancer research 2013;33:5415-20.

[24] Lin Y, Xu T, Zhou S, Cui M. MicroRNA-363 inhibits ovarian cancer progression by inhibiting NOB1. Oncotarget 2017;8:101649-58.

[25] Shi H, Shen H, Xu J, Zhao S, Yao S, Jiang N. MiR-143-3p suppresses the progression of ovarian cancer. American journal of translational research 2018;10:866-74.

[26] Qin A, Zhu J, Liu X, Zeng D, Gu M, Lv C. MicroRNA-1271 inhibits cellular proliferation of hepatocellular carcinoma. Oncology letters 2017;14:6783-8.

[27] Liu H, Wang H, Liu X, Yu T. miR-1271 inhibits migration, invasion and epithelial-mesenchymal transition by targeting ZEB1 and TWIST1 in pancreatic cancer cells. Biochemical and biophysical 
research communications 2016;472:346-52.

[28] Liu X, Ma L, Rao Q, Mao Y, Xin Y, Xu H, et al. MiR-1271 Inhibits Ovarian Cancer Growth by Targeting Cyclin G1. Medical science monitor : international medical journal of experimental and clinical research 2015;21:3152-8.

[29] Xie F, Huang Q, Liu CH, Lin XS, Liu Z, Liu LL, et al. MiR-1271 negatively regulates AKT/MTOR signaling and promotes apoptosis via targeting PDK1 in pancreatic cancer. European review for medical and pharmacological sciences 2018;22:678-86.

[30] Xiang XJ, Deng J, Liu YW, Wan LY, Feng M, Chen J, et al. MiR-1271 Inhibits Cell Proliferation, Invasion and EMT in Gastric Cancer by Targeting FOXQ1. Cellular physiology and biochemistry : international journal of experimental cellular physiology, biochemistry, and pharmacology 2015;36:1382-94.

[31] Trimarchi JM, Lees JA. Sibling rivalry in the E2F family. Nature reviews Molecular cell biology 2002;3:11-20.

[32] Zhao J, Wu XY, Ling XH, Lin ZY, Fu X, Deng YH, et al. Analysis of genetic aberrations on chromosomal region 8q21-24 identifies E2F5 as an oncogene with copy number gain in prostate cancer. Medical oncology 2013;30:465.

[33] Yao YL, Wu XY, Wu JH, Gu T, Chen L, Gu JH, et al. Effects of microRNA-106 on proliferation of gastric cancer cell through regulating p21 and E2F5. Asian Pacific journal of cancer prevention : APJCP 2013;14:2839-43.

[34] Lu G, Sun Y, An S, Xin S, Ren X, Zhang D, et al. MicroRNA-34a targets FMNL2 and E2F5 and suppresses the progression of colorectal cancer. Experimental and molecular pathology 2015;99:173-9.

[35] Umemura S, Shirane M, Takekoshi S, Kusakabe T, Itoh J, Egashira N, et al. Overexpression of E2F-5 correlates with a pathological basal phenotype and a worse clinical outcome. British journal of cancer 2009;100:764-71.

[36] Fang DZ, Wang YP, Liu J, Hui XB, Wang XD, Chen X, et al. MicroRNA-129-3p suppresses tumor growth by targeting E2F5 in glioblastoma. European review for medical and pharmacological sciences 2018;22:1044-50.

[37] Tian H, Hou L, Xiong YM, Huang JX, Zhang WH, Pan YY, et al. miR-132 targeting E2F5 suppresses cell proliferation, invasion, migration in ovarian cancer cells. American journal of translational research 2016;8:1492-501.

[38] Zhang Y, Zhu X, Zhu X, Wu Y, Liu Y, Yao B, et al. MiR-613 suppresses retinoblastoma cell proliferation, invasion, and tumor formation by targeting E2F5. Tumour biology : the journal of the International Society for Oncodevelopmental Biology and Medicine 2017;39:1010428317691674. 


\section{Table}

Table 1 Relationship between miR-1271-5p expression and their clinic-pathological characteristics of OC patients.

\begin{tabular}{|c|c|c|c|c|}
\hline \multirow{2}{*}{ Characteristics } & \multirow{2}{*}{ Cases } & \multicolumn{2}{|c|}{ miR-1271-5p } & \multirow{2}{*}{ P-value } \\
\hline & & High & Low & \\
\hline Age (years) & & & & 0.073 \\
\hline$\geq 60$ & 25 & 11 & 14 & \\
\hline$\square 60$ & 20 & 9 & 11 & \\
\hline Histological grading & & & & 0.118 \\
\hline $1-2$ & 28 & 13 & 15 & \\
\hline 3 & 17 & 7 & 10 & \\
\hline Tumor size & & & & 0.18 \\
\hline$<5 \mathrm{~cm}$ & 30 & 14 & 16 & \\
\hline$\geq 5 \mathrm{~cm}$ & 15 & 6 & 9 & \\
\hline FIGO stage & & & & $0.035 *$ \\
\hline I-II & 33 & 13 & 20 & \\
\hline III-IV & 12 & 7 & 5 & \\
\hline Lymph node metastasis & & & & $0.007^{*}$ \\
\hline No & 35 & 15 & 20 & \\
\hline Yes & 10 & 5 & 5 & \\
\hline
\end{tabular}

Statistical analyses were performed by the $\chi 2$ test.

$* \mathrm{P} \square 0.05$ was considered significant.

\section{Figures}

A

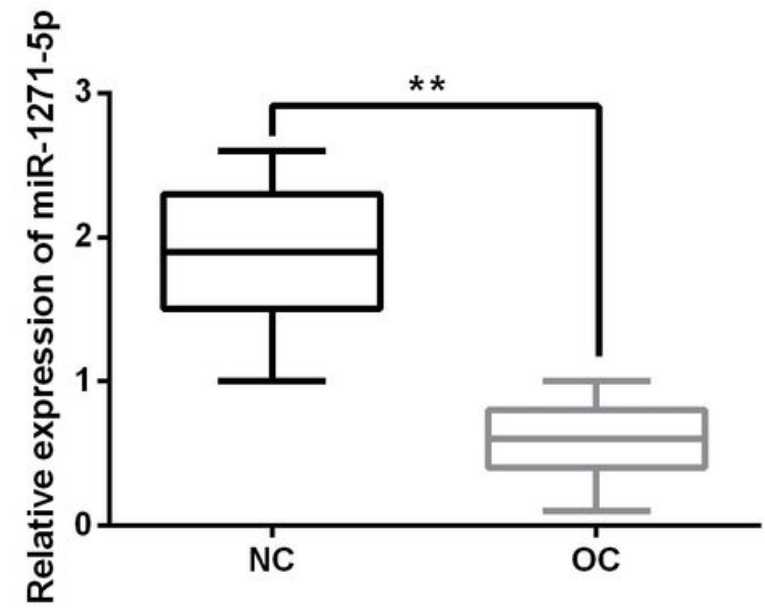

B

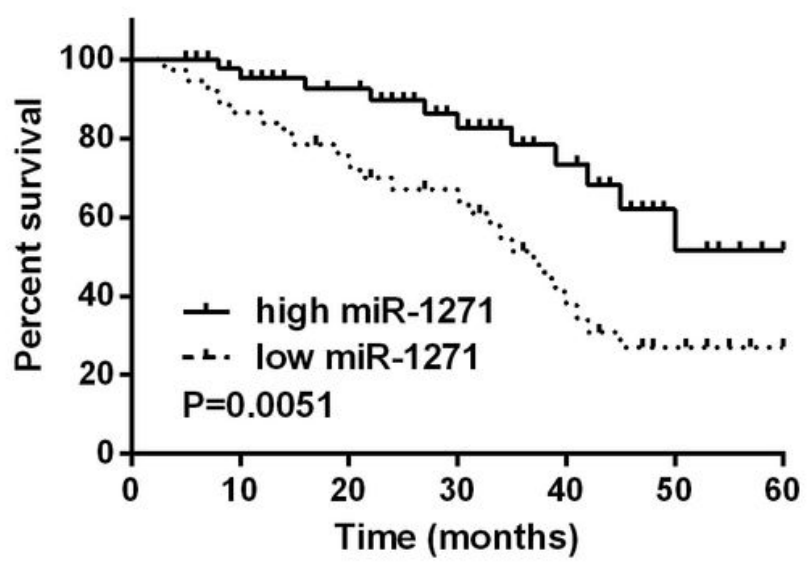


Figure 1

MiR-1271-5p was downregulated in OC tissues. (A) MiR-1271-5p expression in OC and normal tissues (B) Low miR-1271-5p expression was related to shorter overall survival in OC patients. ${ }^{*} \mathrm{P}<0.01$.

A

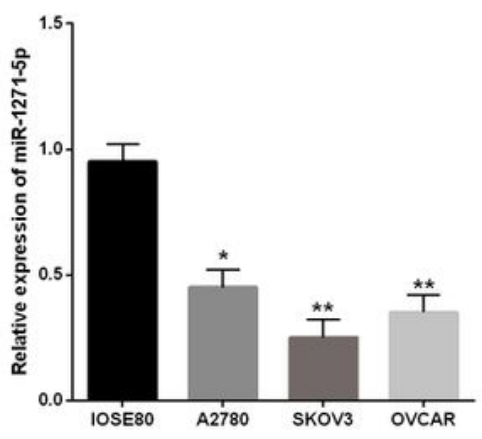

C

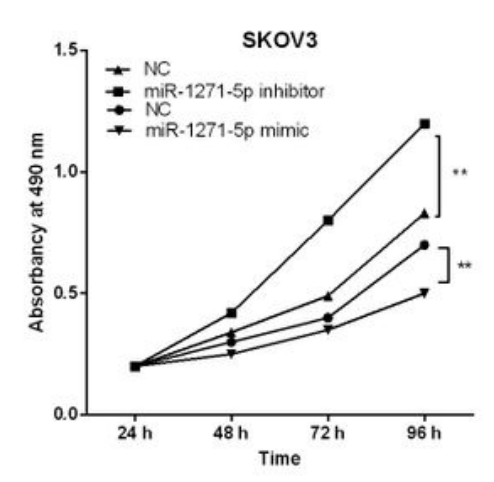

E

Migration

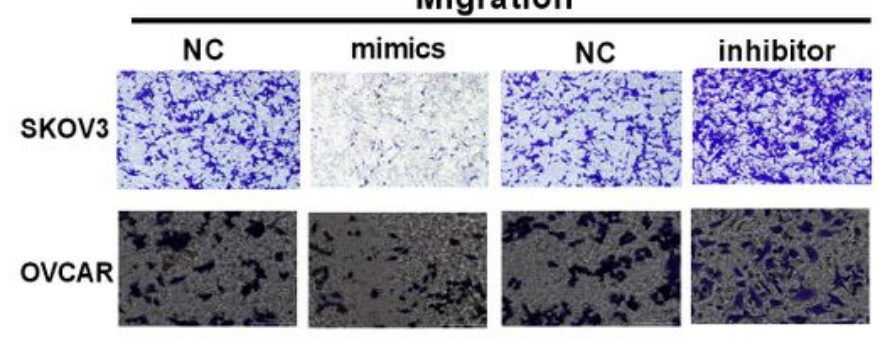

$\mathbf{F}$

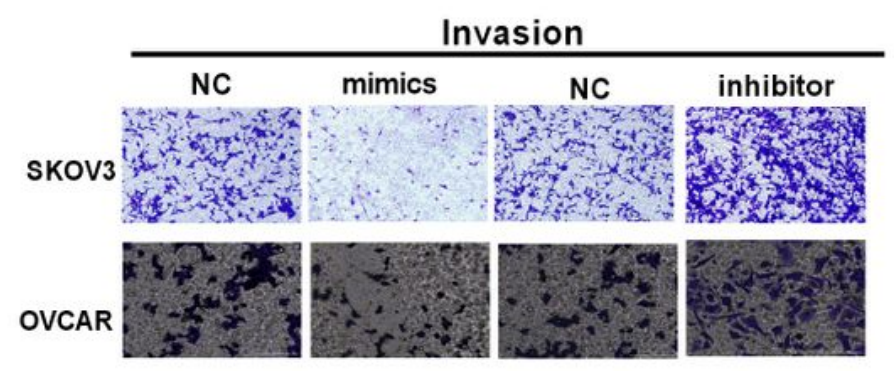

F

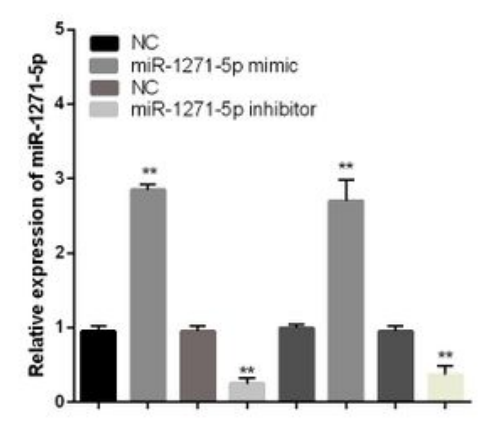

D

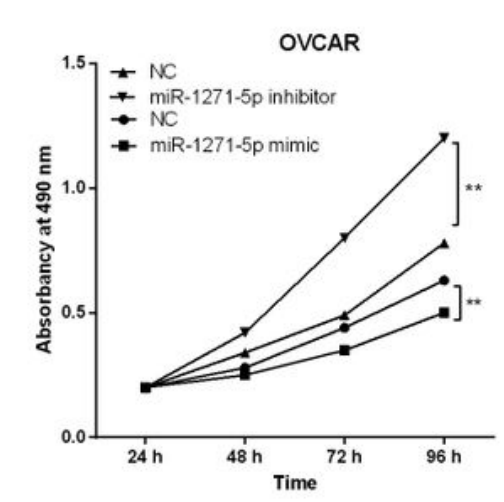

B 
MiR-1271-5p inhibited cell proliferation, migration and invasion in OC. (A) MiR-1271-5p expression in A2780, SKOV3, OVCAR and IOSE80 cells (B) MiR-1271-5p expression in SKOV3 and OVCAR cells containing miR-1271-5p mimics or inhibitor (C, D) Cell proliferation in SKOV3 and OVCAR cells with miR1271-5p mimics or inhibitor ( $E, F)$ MiR-1271-5p mimics or inhibitor regulated cell migration and invasion * $\mathrm{P}<0.05$, ** $\mathrm{P}<0.01$.
A
MiR-1271-5p
3'...GUUU GUUUUUAAAAU GU GCCAAU....5'
|||||| $\mid$
Wild of E $2 F 5$ 3'-UTR
5....ACUCACGAACGAUCCACGGUU C...3'
Mutant of E2F5 3'-UTR
5'.... ACU CACGAACGAU CGU GCCAAC....3'

B
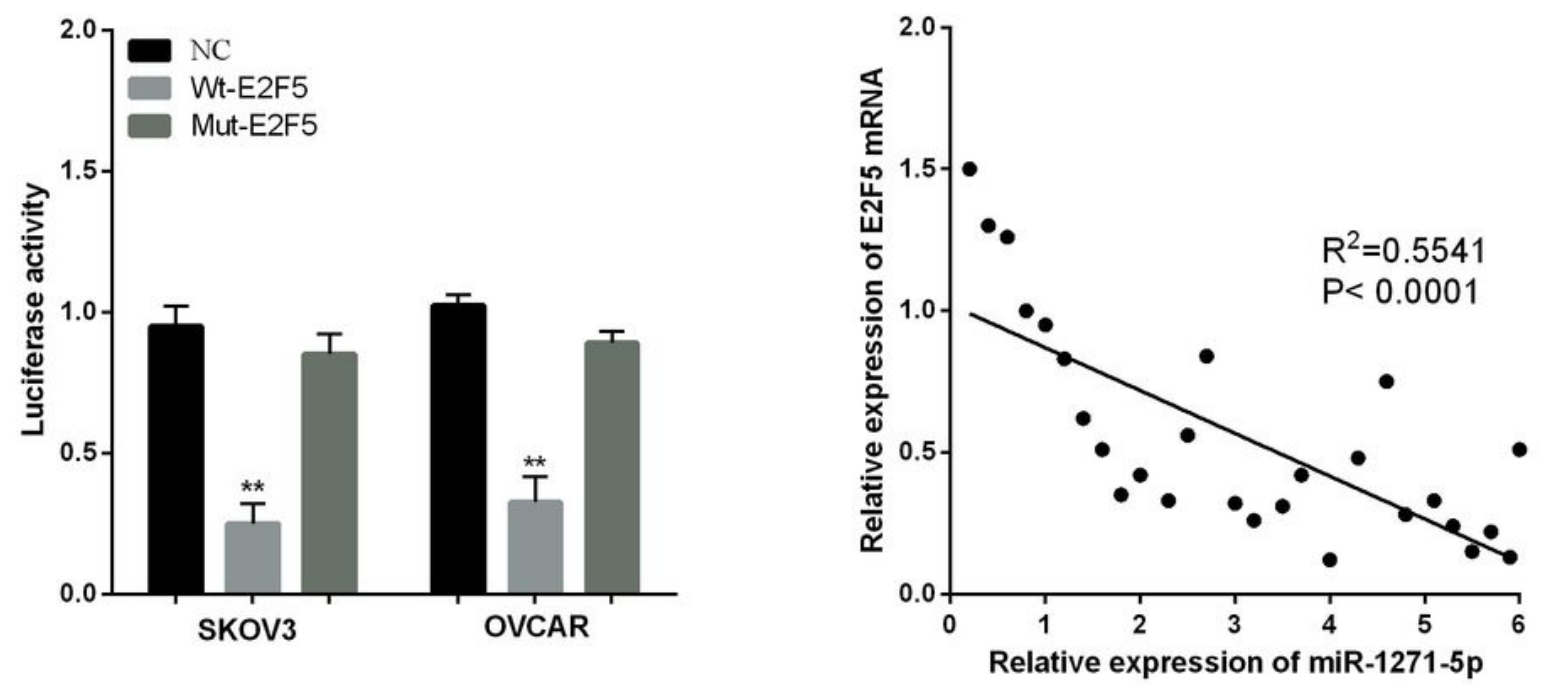

D

E
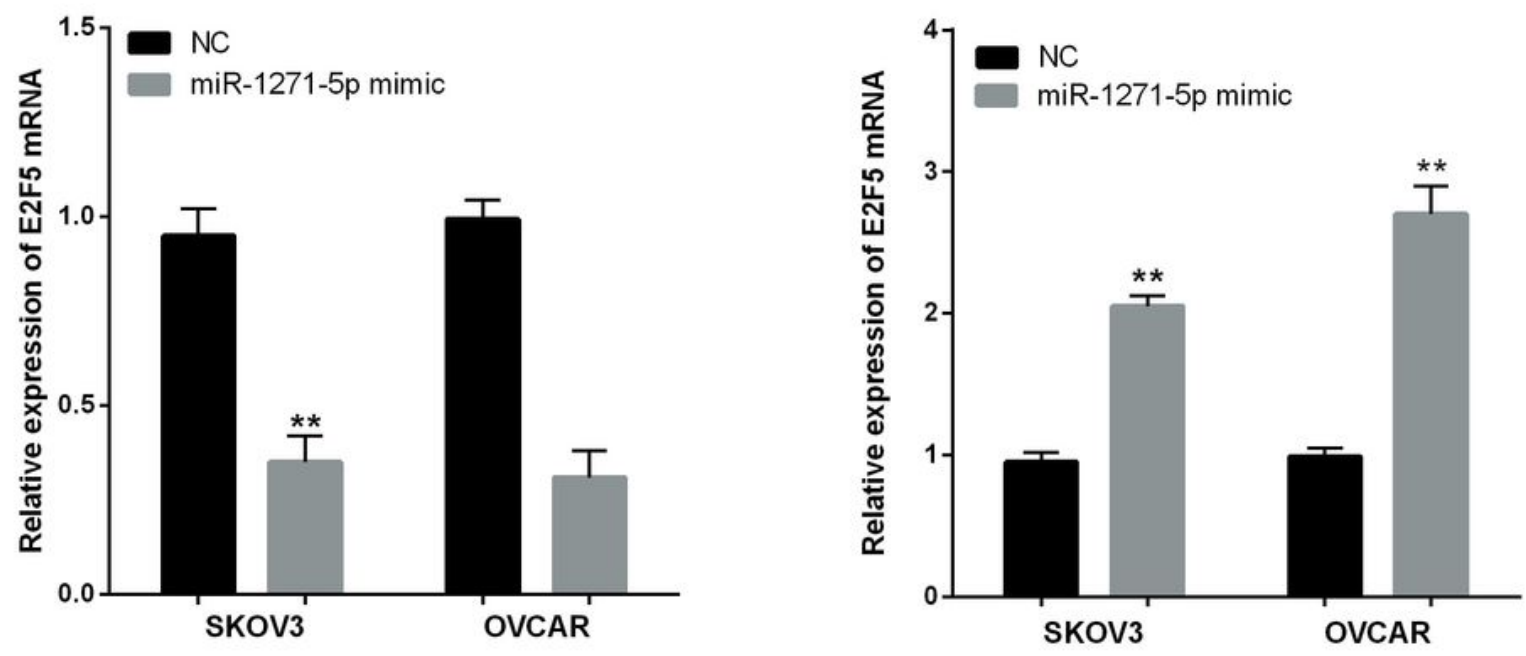

Figure 3 
E2F5 was a direct target of miR-1271-5p in OC cells. (A) The binding sites between miR-1271-5p and E2F5 (B) Luciferase reporter assay (C) MiR-1271-5p inversely regulated E2F5 expression (D, E) E2F5 expression was in SKOV3 and OVCAR cells containing miR-1271-5p mimics or inhibitor ** $\mathrm{P}<0.01$.

A

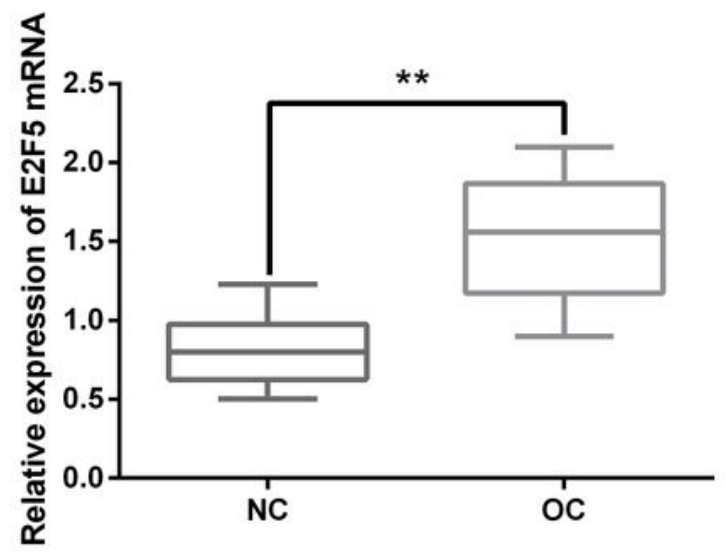

B

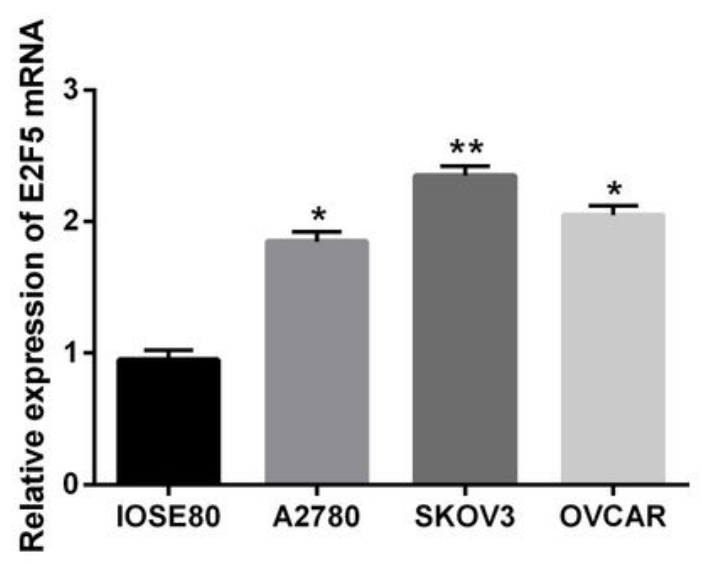

C

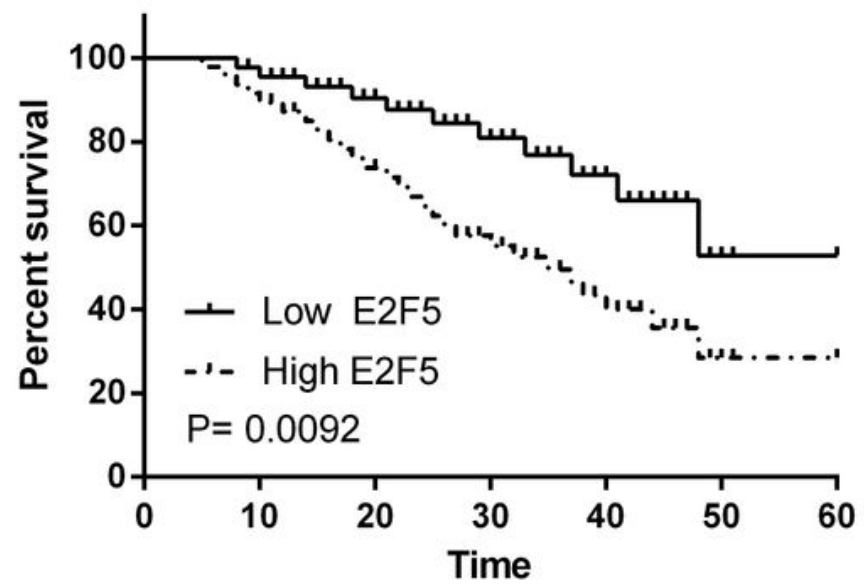

Figure 4

Upregulation of E2F5 was detected in OC. (A) E2F5 expression in OC tissues and normal tissues (B) E2F5 expression in A2780, SKOV3, OVCAR and IOSE80 cell lines. (C) Shorter overall survival in OC patients with 
A

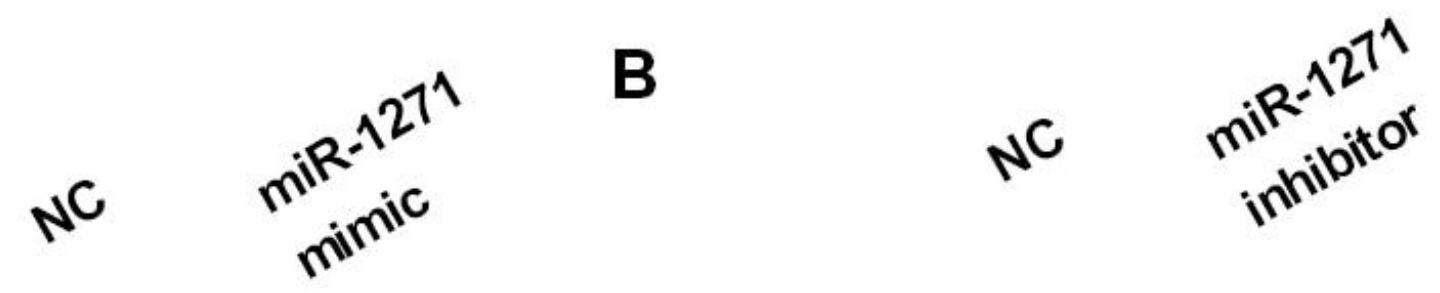

\section{E-cadherin}

N-cadherin

Vimentin

\section{E2F5}

mTOR

p-mTOR

\section{GADPH}

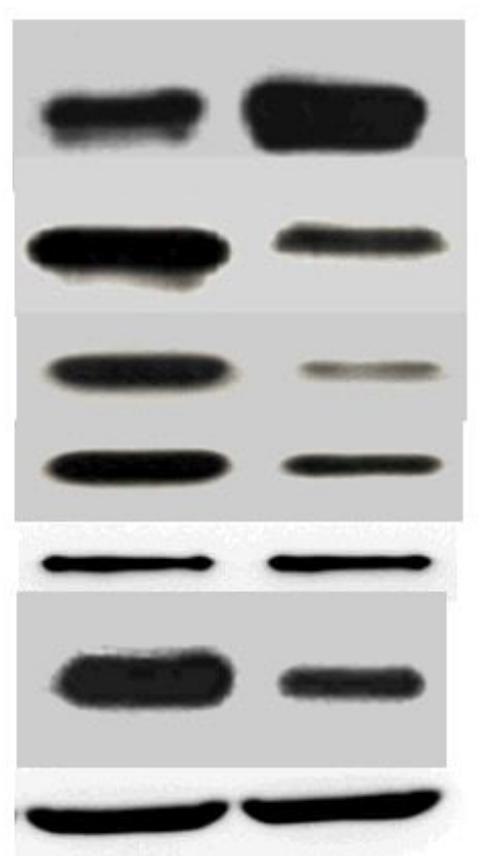

E-cadherin

N-cadherin

Vimentin

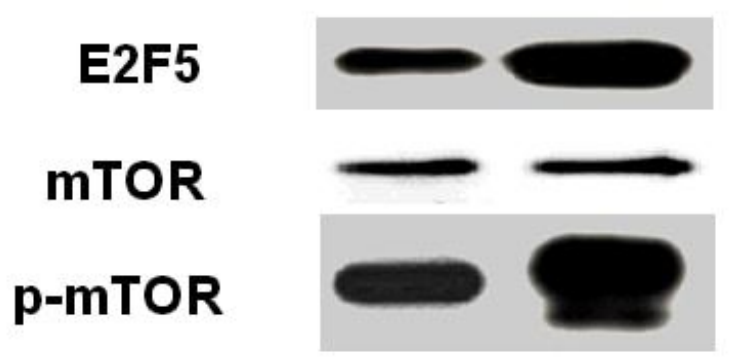

GADPH
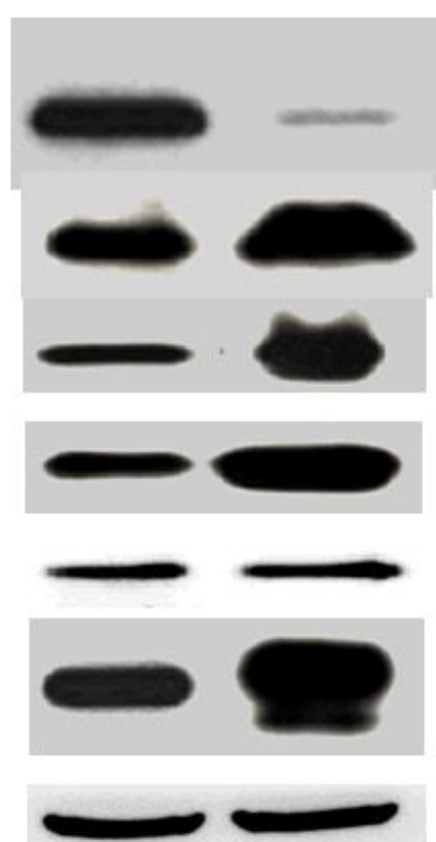

Figure 5

MiR-1271-5p negatively regulated EMT and mTOR pathway in OC. (A, B) The protein expression of Vimentin, N-cadherin, E-cadherin, mTOR and p-mTOR in SKOV3 cells containing miR-1271-5p mimics or inhibitor. 